\title{
The proliferation marker Chromatin Assembly Factor-1 is of clinical value in predicting the biological behaviour of salivary gland tumours
}

\author{
STEFANIA STAIBANO ${ }^{1}$, MASSIMO MASCOLO $^{1}$, ALBA ROCCO $^{2}$, LORENZO LO MUZIO ${ }^{4,6}$, \\ GENNARO ILARDI $^{1}$, MARIA SIANO ${ }^{1}$, GIUSEPPE PANNONE ${ }^{5}$, MARIA LUISA VECCHIONE ${ }^{1}$, \\ LOREDANA NUGNES ${ }^{1}$, LUIGI CALIFANO ${ }^{3}$, ROSANNA ZAMPARESE ${ }^{7}$, \\ PANTALEO BUFO 5 and GAETANO DE ROSA ${ }^{1,6}$
}

\begin{abstract}
Departments of ${ }^{1}$ Biomorphological and Functional Sciences, Pathology Section, ${ }^{2}$ Clinical and Experimental Medicine, Gastroenterology Unit and ${ }^{3}$ Maxillofacial Surgery, University 'Federico II' of Naples, School of Medicine, via Pansini 5, I-80131, Naples; Departments of ${ }^{4}$ Surgical Sciences, Section of Dental Sciences and ${ }^{5}$ Surgical Sciences,

Pathology Section, University of Foggia, School of Medicine, via Carelli 28, I-71100, Foggia;

${ }^{6}$ Centro di Riferimento Oncologico di Basilicata (C.R.O.B.), I.R.C.C.S., via Padre Pio 1,

Rionero in Vulture (PZ); ${ }^{7}$ Department of Surgical Sciences, Pathology Section,

General Hospital ‘C.G. Mazzoni’, via degli Iris, I-63100, Ascoli Piceno, Italy
\end{abstract}

Received August 19, 2010; Accepted September 17, 2010

DOI: 10.3892/or_00001036

\begin{abstract}
Salivary gland tumours (SGT) constitute a diagnostically challenging group of neoplasms with frequently unpredictable clinical outcome. The proliferation rate facilitates the identification of aggressive SGT. The Chromatin Assembly Factor-1 (CAF-1) is a major epigenetic regulator of nuclear chromatin organization during DNA replication. It plays a critical function in human tumourigenesis and has been proposed as a new proliferation and prognostic marker for some malignancies. This study focused on the role of CAF-1/p60 protein as a marker of clinical value for SGT. The expression of CAF-1/p60 was evaluated by immunohistochemistry on a retrospective series of 362 surgically excised benign and malignant SGT with different histogenesis and, when available, on fine-needle pre-surgical cytological biopsies. The resulting data were compared with traditional prognostic parameters, including the expression of the routine proliferation marker ki67/MIB1. CAF-1/p60 was detectable in all SGT, with highest degree of expression in metastasizing malignant tumours. Moreover, the cases of benign tumours which progressed to carcinoma during the follow-up, showed significantly higher $\mathrm{CAF}-1 / \mathrm{p} 60$ expression than non-
\end{abstract}

Correspondence to: Dr Stefania Staibano, Department of Biomorphological and Functional Sciences, Oral and Facial Pathology Section, School of Medicine, University 'Federico II' of Naples, via Pansini 5, I-80131, Naples, Italy

E-mail: staibano@unina.it

Key words: Chromatin Assembly Factor-1, salivary gland tumours, aggressive behaviour progressing benign SGT, both on histological sections and cytological smears of the primary tumour. Cox's multiple regression analysis selected CAF-1/p60 expression as the best independent predictor of cancer development for benign SGT $(\mathrm{p}<0.0001)$, and the best independent predictor of metastasis onset for malignant tumours $(\mathrm{p}<0.0004)$. Overexpression of CAF-1/p60, on histological and/or cytological samples, characterizes malignant SGT with aggressive behaviour, irrespective of their specific histotype, and allows the early diagnosis of progression toward malignancy of morphologically benign tumours.

\section{Introduction}

Salivary gland tumours (SGT) constitute morphologically and biologically heterogeneous neoplasms, which give rise to significant diagnostic and management challenges (1-3). These tumours are rare, with an overall incidence of 0.5 3.0 cases per 100000 per year. Malignant SGT accounts for about $0.5 \%$ of all malignancies and for $3-6 \%$ of head and neck cancers (1).

Many studies attempted to better define the prognosis of these tumours (4-6). However, the available markers are unable to ultimately discriminate, among benign SGT, the cases that will give rise to progression toward malignancy, and, for malignant SGT, the cases with a metastasizing behaviour.

Cell proliferation rate has been proposed as an useful adjunctive tool for predicting the outcome of SGT, but there is not a definitive agreement between researchers on the best proliferation-associated prognostic marker for these tumours $(3,4,6-12)$.

The orderly progression of the cell cycle is governed by epigenetic modifications, mostly concerning the hierarchical assembly and remodelling of chromatin, which are regulated 
Table I. Clinical and pathological features, CAF-1/p60 and ki67/MIB1 immunohistochemical expression in 268 cases of benign SGT.

\begin{tabular}{|c|c|c|c|c|c|c|c|}
\hline \multirow[t]{2}{*}{ Characteristics } & \multirow[t]{2}{*}{ Total } & \multirow[t]{2}{*}{$(\%)$} & \multicolumn{2}{|c|}{ CAF-1/p60 (quickscore) } & \multicolumn{3}{|c|}{ ki67/MIB1 (quickscore) } \\
\hline & & & $5(2+3)$ & $6(3+3)$ & $3(1+2)$ & $4(2+2)$ & $5(2+3)$ \\
\hline \multicolumn{8}{|l|}{ No. of subjects } \\
\hline Male & 116 & 43.28 & $114(42.53)$ & $2(0.75)$ & $100(37.31)$ & $8(2.99)$ & $8(2.99)$ \\
\hline Female & 152 & 56.72 & $150(55.97)$ & $2(0.75)$ & $131(48.69)$ & $12(4.48)$ & $9(3.36)$ \\
\hline \multicolumn{8}{|l|}{ Age } \\
\hline Average (range) & 39.83 & $(9-80)$ & - & - & - & - & - \\
\hline \multicolumn{8}{|l|}{ Disease site } \\
\hline Parotid gland & 203 & 75.75 & $200(74.63)$ & $3(1.12)$ & $180(67.16)$ & $12(4.48)$ & $11(4.11)$ \\
\hline Submandibular gland & 33 & 12.31 & $33(12.31)$ & 0 & $30(11.20)$ & $2(0.75)$ & $1(0.37)$ \\
\hline Sublingual gland & 1 & 0.4 & $1 \quad(0.37)$ & 0 & $1 \quad(0.37)$ & 0 & 0 \\
\hline Minor salivary gland & 31 & 11.57 & $30(11.20)$ & $1(0.37)$ & $20 \quad(7.46)$ & $6(2.23)$ & $5(1.87)$ \\
\hline \multicolumn{8}{|l|}{ Histotype } \\
\hline $\mathrm{BCA}$ & 3 & 1.12 & $3 \quad(1.12)$ & 0 & $3 \quad(1.12)$ & 0 & 0 \\
\hline PA & 261 & 97.39 & $258(96.27)$ & $3(1.12)$ & $226(84.33)$ & $19(7.09)$ & $16(5.97)$ \\
\hline Atypical PA & 4 & 1.49 & $3 \quad(1.12)$ & $1(0.37)$ & $2 \quad(0.75)$ & $1(0.37)$ & $1(0.37)$ \\
\hline \multicolumn{8}{|c|}{ Evolution to carcinoma ex-PA } \\
\hline No & 263 & 98.13 & $263(98.14)$ & 0 & $230(85.82)$ & $18(6.72)$ & $15(5.59)$ \\
\hline Yes & 5 & 1.87 & $1 \quad(0.37)$ & $4(1.49)$ & $1 \quad(0.37)$ & $2(0.75)$ & $2(0.75)$ \\
\hline
\end{tabular}

by various Assembly Factors $(13,14)$. Among these, the Chromatin Assembly Factor-1 (CAF-1), a heterotrimeric complex, composed of the histone-chaperone proteins $\mathrm{p} 48$, p60 and p150, is the most powerful discriminator between the proliferative and the quiescent state (15-17). CAF-1/p60 has been recently proposed as a new sensitive marker of cell proliferation, able to predict the prognosis of some human cancer types (18-20).

We have performed a study on a retrospective series of benign and malignant SGT, with the aim to establish whether the immunohistochemical expression of CAF-1/p60 protein may have a value in discriminating SGT with different clinical behaviour, beyond the available prognostic parameters including the routinely assessed proliferation marker ki67/MIB1.

\section{Materials and methods}

Selection of cases. The study population was selected among the patients diagnosed with SGT at the Federico II University of Naples, Italy, from April 1993 to March 2008.

Only the cases of patients treated primarily with surgery, with healthy surgical margins distant from the tumour by at least $1 \mathrm{~cm}$, and with clinical follow-up data not less than 12 months, were considered for the study.

Patients characteristics, the anatomic site and histotype of primary tumours and, for malignant SGT, the degree of differentiation and tumour stage were collected and recorded in a data base $(21,22)$.
Immunohistochemistry. Immunohistochemical analysis was performed on 4- $\mu \mathrm{m}$ thick serial sections, mounted on poly-Llysine coated glass slides. Sections were deparaffinized, and underwent antigen retrieval by microwave oven treatment ( 5 min $\times 3$ times, in $1 \%$ sodium citrate buffer, $\mathrm{pH}$ 6.0); nonspecific bindings were blocked by incubation $(2 \mathrm{~h}$ at room temperature) with $1.5 \%$ non-immune mouse serum (1:20, Dakopatts, Hamburg, Germany). Endogenous peroxidases were quenched with $0.3 \%$ hydrogen peroxide in methanol; slides were rinsed twice with Tris- $\mathrm{HCl}$ buffer, and incubated overnight at $4^{\circ} \mathrm{C}$ with anti-CAF-1/p60 antibody (SS53ab8133, Abcam, Cambridge, MA, USA, diluted 1:300) and ki67/MIB1 antibody (MIB1, Dako A/S, Glostrup, Denmark, diluted 1:200) $(19,20,23)$.

The standard streptavidin-biotin linked horseradish peroxidase (LSAB) technique using the Dako (LSAB kit HRP, Carpinteria, CA) was then performed, with the 3,3'diaminobenzidine (DAB, Vector Laboratories Inc., Burlingame, CA, USA) being used as a substrate chromogen solution for the development of the peroxidase activity. After nuclear counterstaining with Mayer's haematoxylin for $30 \mathrm{sec}$, sections were mounted and cover-slipped with a synthetic medium.

For each run, positive controls were performed on sections of breast carcinomas for CAF-1/p60, and on small bowel samples for ki67/MIB1. For negative controls, nonimmune serum in TBS buffer (1:500) was used instead of the two primary antibodies. The cells with a definite brown nuclear staining were judged as positive for both the anti- 

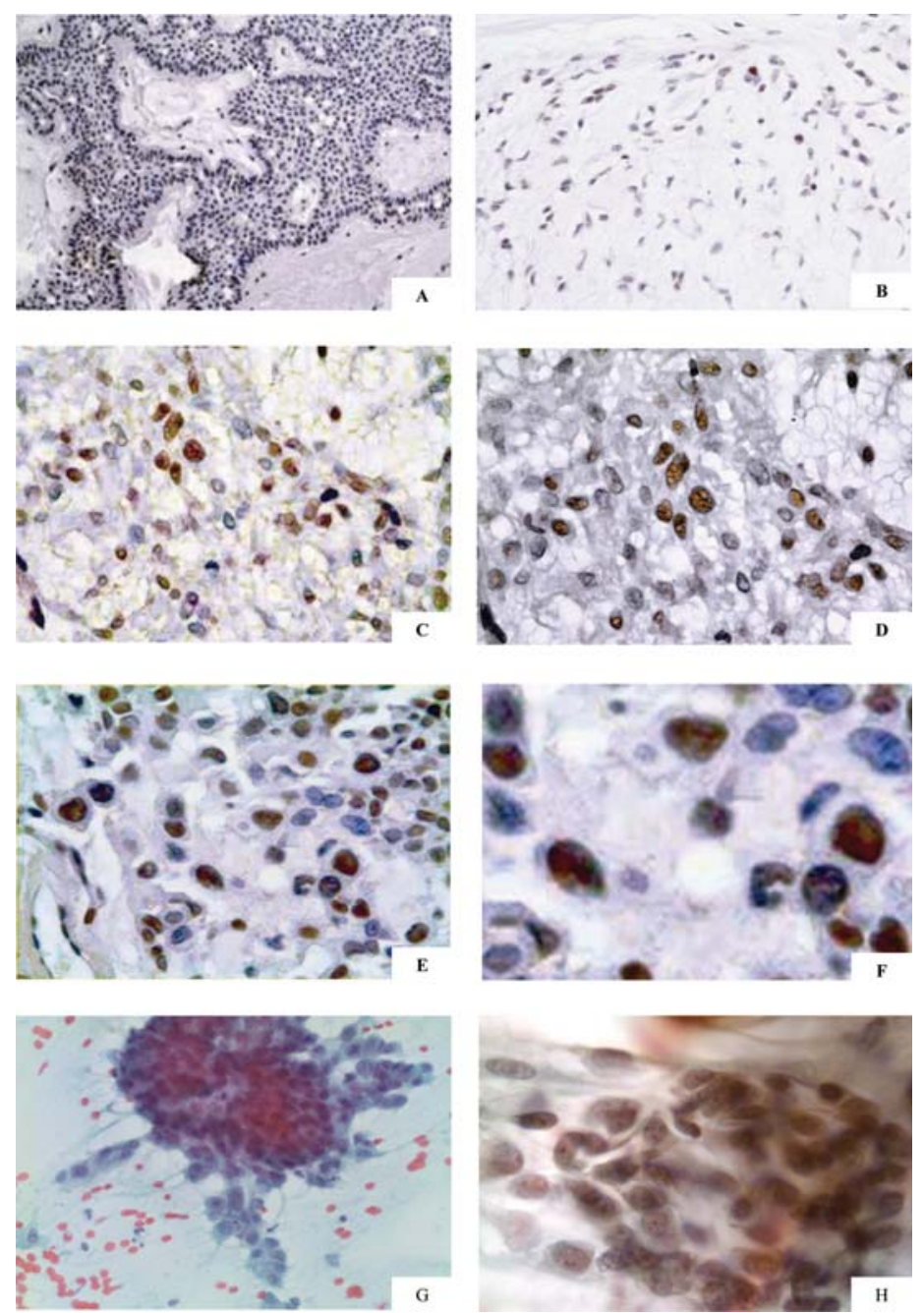

Figure 1. Immunohistochemical expression of CAF-1/p60 in benign SGT (LSAB-HRP, nuclear counterstaining with haematoxylin). Focal and weak nuclear immunostaining for CAF-1/p60 in BCA (A, x106) and PA (B, x150). Higher expression of CAF-1/p60 in two cases of PA (ordinary type: C, x200; D, x250 and atypical: E, x250; F, x400) which progressed to CXPA during follow-up; PA progressed to CXPA (PAP-stained FNAB: G, x250; CAF-1/p60 immunostaining: $\mathrm{H}, \mathrm{x} 400)$.

bodies. The immunohistochemical expression was evaluated semi-quantitatively and scored according to the "quickscore" method, which takes into consideration either the percentage of immunostained neoplastic cells throughout the entire tumour section (scores 1-6: 1, 0-4\%; 2, 5-19\%; 3, 20-39\%; 4, 40$59 \% ; 5,60-79 \% ; 6,80-100 \%)$, termed 'category A', and the staining intensity (scores $0-3$, corresponding to negative, weak, intermediate and strong staining), termed 'category B' (24).

The evaluation was performed independently by two expert pathologists (S.S. and M.M.), unaware of patients' clinical status. Discordant cases were discussed and resolved by consensus.

For 23 cases of benign tumours, pre-surgical PAP-stained slides of the fine-needle aspiration biopsy (FNAB) cytological samples, used for morphological diagnosis, were also available, and were re-used for the immunostaining with CAF-1/p60 protein, according to the technique described in literature (Table I) (25).

Statistical analysis. Data were analyzed with SPSS package for Windows (release 13.0). The intra- and inter-observer agreement of the evaluation of the CAF-1/p60 and ki67/
MIB1 quickscore was calculated by k-statistics: a K coefficient $>0.75$ indicated excellent reproducibility; a K-value between 0.4 and 0.75 denoted moderate reproducibility, and a K-value $<0.4$ denoted marginal or poor reproducibility (26).

To evaluate the prognostic value of CAF-1/p60 expression with respect to conventional parameters and ki67/MIB1 expression, a univariate analysis was performed by KaplanMeier plots for each variable. The significant level was set at $\mathrm{p}<0.05$. To verify whether variables were univariately related to our end-points (development of malignant tumour in patients with benign cancer baseline and disease-free interval in patients with malignant tumours baseline) curves were compared by the log-rank test or the t-test for trend, when two or three curves had to be compared, respectively.

In order to establish whether CAF-1/p60 expression contributed to a better definition of the prognosis of patients, after the conventional data had been taken into account, a multiple regression analysis according to the Cox's proportional hazard model was performed.

The clinical usefulness of prognostic indices predicting the risk for cancer development in patients who had a 
Table II. Clinical and pathological features, CAF-1/p60 and ki67/MIB1 immunohistochemical expression in 94 cases of malignant SGT.

\begin{tabular}{|c|c|c|c|c|c|c|c|c|}
\hline $\begin{array}{c}\text { Patient } \\
\text { no. }\end{array}$ & Gender & Age & Diagnosis & Gland & $\begin{array}{l}\text { CAF-1/p60 } \\
\text { (quickscore) }\end{array}$ & $\begin{array}{l}\text { ki67/MIB1 } \\
\text { (quickscore) }\end{array}$ & F-UP & Stage \\
\hline 1 & M & 49 & PLGC & Minor salivary gland & $6(3+3)$ & $3(1+2)$ & 26 & pT1N0M0 \\
\hline 2 & $\mathrm{~F}$ & 54 & PLGC & Parotid & $8(5+3)$ & $3(1+2)$ & $60 \mathrm{R}(34)$ & pT1NxM0 \\
\hline 3 & $\mathrm{~F}$ & 13 & $\mathrm{AC}$ & Parotid & $7(4+3)$ & $4(2+2)$ & 74 & pT1NxM0 \\
\hline 4 & $\mathrm{~F}$ & 33 & $\mathrm{AC}$ & Parotid & $6(3+3)$ & $4(2+2)$ & 67 & pT3N0M0 \\
\hline 5 & M & 45 & $\mathrm{AC}$ & Parotid & $7(4+3)$ & $4(2+2)$ & 70 & pT2NxM0 \\
\hline 6 & $\mathrm{~F}$ & 47 & $\mathrm{AC}$ & Parotid & $6(3+3)$ & $4(2+2)$ & 42 & pT1NxM0 \\
\hline 7 & $\mathrm{~F}$ & 78 & $\mathrm{AC}$ & Minor salivary gland & $7(4+3)$ & $4(2+2)$ & 44 & pT3NxM0 \\
\hline 8 & F & 41 & $\mathrm{AC}$ & Parotid & $7(4+3)$ & $4(2+2)$ & 20 & pT1N0M0 \\
\hline 9 & $\mathrm{~F}$ & 57 & $\mathrm{AC}$ & Parotid & $6(3+3)$ & $4(2+2)$ & 93 & pT1NxM0 \\
\hline 10 & M & 52 & $\mathrm{AC}$ & Parotid & $8(5+3)$ & $4(2+2)$ & $150 \mathrm{R}(40,142)$ & pT1NxM0 \\
\hline 11 & M & 55 & $\mathrm{AC}$ & Parotid & $6(3+3)$ & $4(2+2)$ & 158 & pT1N0M0 \\
\hline 12 & $\mathrm{~F}$ & 19 & $\mathrm{AC}$ & Submandibolar gland & $6(3+3)$ & $4(2+2)$ & 142 & pT1NxM0 \\
\hline 13 & $\mathrm{~F}$ & 57 & $\mathrm{AC}$ & Parotid & $6(3+3)$ & $4(2+2)$ & 180 & pT2NxM0 \\
\hline 14 & M & 50 & $\mathrm{AC}$ & Parotid & $6(3+3)$ & $4(2+2)$ & 110 & pT2NxM0 \\
\hline 15 & $\mathrm{~F}$ & 17 & $\mathrm{AC}$ & Parotid & $7(4+3)$ & $4(2+2)$ & 167 & pT1NxM0 \\
\hline 16 & M & 60 & $\mathrm{AC}$ & Parotid & $7(4+3)$ & $4(2+2)$ & 192 & pT2NxM0 \\
\hline 17 & $\mathrm{~F}$ & 23 & $\mathrm{ACC}$ & Submandibolar gland & $7(4+3)$ & $4(2+2)$ & 97 & pT1NxM0 \\
\hline 18 & $\mathrm{~F}$ & 34 & ACC & Minor salivary gland & $7(4+3)$ & $5(2+3)$ & 82 & pT1NxM0 \\
\hline 19 & $\mathrm{~F}$ & 39 & $\mathrm{ACC}$ & Parotid & $6(3+3)$ & $6(3+3)$ & 29 & pT2N0M0 \\
\hline 20 & $\mathrm{~F}$ & 41 & $\mathrm{ACC}$ & Submandibolar gland & $7(4+3)$ & $6(3+3)$ & 54 & pT3NOMO \\
\hline 21 & M & 48 & ACC & Minor salivary gland & $9(6+3)$ & $6(3+3)$ & 71 M (58) R (33) & pT4aNxM0 \\
\hline 22 & $\mathrm{~F}$ & 56 & $\mathrm{ACC}$ & Minor salivary gland & $8(5+3)$ & $6(3+3)$ & 34 & pT4bNxM1 \\
\hline 23 & F & 63 & $\mathrm{ACC}$ & Minor salivary gland & $7(4+3)$ & $4(2+2)$ & 44 & pT2NxM0 \\
\hline 24 & $\mathrm{~F}$ & 41 & $\mathrm{ACC}$ & Submandibolar gland & $6(3+3)$ & $4(2+2)$ & 141 & pT3N0M0 \\
\hline 25 & $\mathrm{~F}$ & 74 & $\mathrm{ACC}$ & Minor salivary gland & $6(3+3)$ & $4(2+2)$ & 181 & pT2NxM0 \\
\hline 26 & $\mathrm{~F}$ & 49 & $\mathrm{ACC}$ & Submandibolar gland & $7(4+3)$ & $5(2+3)$ & 12 & pT2N0M0 \\
\hline 27 & $\mathrm{~F}$ & 49 & $\mathrm{ACC}$ & Minor salivary gland & $7(4+3)$ & $6(3+3)$ & 173 & pT2NxM0 \\
\hline 28 & $\mathrm{~F}$ & 69 & $\mathrm{ACC}$ & Minor salivary gland & $7(4+3)$ & $6(3+3)$ & 84 & pT2NxM0 \\
\hline 29 & $\mathrm{~F}$ & 48 & $\mathrm{ACC}$ & Minor salivary gland & $8(5+3)$ & $6(3+3)$ & $193 \mathrm{R}(61)$ & pT1NxM0 \\
\hline 30 & M & 63 & $\mathrm{ACC}$ & Minor salivary gland & $6(3+3)$ & $5(2+3)$ & 41 & pT4aNxM0 \\
\hline 31 & M & 49 & $\mathrm{ACC}$ & Minor salivary gland & $6(3+3)$ & $6(3+3)$ & 170 & pT1NxM0 \\
\hline 32 & M & 59 & $\mathrm{ACC}$ & Minor salivary gland & $8(5+3)$ & $5(2+3)$ & 185 & pT2NOM0 \\
\hline 33 & M & 69 & $\mathrm{ACC}$ & Minor salivary gland & $6(3+3)$ & $6(3+3)$ & 16 & pT1NxM0 \\
\hline 34 & $\mathrm{~F}$ & 56 & $\mathrm{ACC}$ & Submandibolar gland & $7(4+3)$ & $4(2+2)$ & 177 & pT2NxM0 \\
\hline 35 & $\mathrm{~F}$ & 69 & $\mathrm{ACC}$ & Minor salivary gland & $7(4+3)$ & $4(2+2)$ & 181 & pT1NxM0 \\
\hline 36 & M & 50 & $\mathrm{ACC}$ & Parotid & $8(5+3)$ & $4(2+2)$ & 177 & pT3N2bM0 \\
\hline 37 & $\mathrm{~F}$ & 33 & $\mathrm{ACC}$ & Minor salivary gland & $6(3+3)$ & $4(2+2)$ & 34 & pT1NxM0 \\
\hline 38 & $\mathrm{~F}$ & 20 & Low-grade MEC & Parotid & $6(3+3)$ & $4(2+2)$ & 49 & pT1N0M0 \\
\hline 39 & M & 34 & Low-grade MEC & Parotid & $7(4+3)$ & $5(2+3)$ & 137 & pT1N1M0 \\
\hline 40 & $\mathrm{~F}$ & 57 & Low-grade MEC & Minor salivary gland & $7(4+3)$ & $5(2+3)$ & 173 & pT3N0M0 \\
\hline 41 & M & 14 & Low-grade MEC & Minor salivary gland & $6(3+3)$ & $5(2+3)$ & 128 & pT1NxM0 \\
\hline 42 & F & 18 & Low-grade MEC & Parotid & $6(3+3)$ & $4(2+2)$ & 92 & pT2N0M0 \\
\hline 43 & M & 37 & Low-grade MEC & Parotid & $6(3+3)$ & $4(2+2)$ & 129 & pT1NxM0 \\
\hline 44 & $\mathrm{~F}$ & 38 & Low-grade MEC & Parotid & $6(3+3)$ & $4(2+2)$ & 57 & pT1N0M0 \\
\hline 45 & $\mathrm{~F}$ & 40 & Low-grade MEC & Parotid & $6(3+3)$ & $4(2+2)$ & 69 & pT4aN0M0 \\
\hline 46 & $\mathrm{~F}$ & 42 & Low-grade MEC & Parotid & $7(4+3)$ & $4(2+2)$ & 127 & pT1NxM0 \\
\hline 47 & M & 55 & Low-grade MEC & Minor salivary gland & $7(4+3)$ & $4(2+2)$ & 107 & pT1NxM0 \\
\hline 48 & $\mathrm{~F}$ & 62 & Low-grade MEC & Minor salivary gland & $7(4+3)$ & $5(2+3)$ & 103 & pT3NxM0 \\
\hline 49 & M & 74 & Low-grade MEC & Minor salivary gland & $6(3+3)$ & $5(2+3)$ & 129 & pT1NxM0 \\
\hline 50 & M & 63 & Low-grade MEC & Minor salivary gland & $6(3+3)$ & $5(2+3)$ & 33 & pT1NxM0 \\
\hline 51 & $\mathrm{~F}$ & 60 & Low-grade MEC & Minor salivary gland & $7(4+3)$ & $5(2+3)$ & 12 & pT1NxM0 \\
\hline 52 & $\mathrm{~F}$ & 49 & Low-grade MEC & Minor salivary gland & $7(4+3)$ & $5(2+3)$ & 12 & pT1NxM0 \\
\hline 53 & $\mathrm{~F}$ & 42 & Low-grade MEC & Parotid & $6(3+3)$ & $5(2+3)$ & 128 & pT1NxM0 \\
\hline
\end{tabular}


Table II. Continued.

\begin{tabular}{|c|c|c|c|c|c|c|c|c|}
\hline $\begin{array}{l}\text { Patient } \\
\text { no. }\end{array}$ & Gender & Age & Diagnosis & Gland & $\begin{array}{l}\text { CAF-1/p60 } \\
\text { (quickscore) }\end{array}$ & $\begin{array}{l}\text { ki67/MIB1 } \\
\text { (quickscore) }\end{array}$ & F-UP & Stage \\
\hline 54 & $\mathrm{~F}$ & 40 & Low-grade MEC & Minor salivary gland & $7(4+3)$ & $5(2+3)$ & 162 & pT2NxM0 \\
\hline 55 & M & 39 & Low-grade MEC & Minor salivary gland & $6(3+3)$ & $5(2+3)$ & 199 & pT1NxM0 \\
\hline 56 & $\mathrm{~F}$ & 37 & Low-grade MEC & Minor salivary gland & $6(3+3)$ & $5(2+3)$ & 20 & pT1NxM0 \\
\hline 57 & M & 49 & Low-grade MEC & Parotid & $7(4+3)$ & $5(2+3)$ & 96 & pT1NxM0 \\
\hline 58 & $\mathrm{~F}$ & 64 & Low-grade MEC & Minor salivary gland & $7(4+3)$ & $5(2+3)$ & 18 & pT1NxM0 \\
\hline 59 & $\mathrm{~F}$ & 33 & Low-grade MEC & Minor salivary gland & $7(4+3)$ & $5(2+3)$ & 197 & pT1NxM0 \\
\hline 60 & M & 39 & Intermediate-grade MEC & Parotid & $8(5+3)$ & $5(2+3)$ & $140 \mathrm{R}(48)$ & pT2N0M0 \\
\hline 61 & $\mathrm{~F}$ & 51 & Intermediate-grade MEC & Minor salivary gland & $9(6+3)$ & $6(3+3)$ & $132 \mathrm{M}(16)$ & pT2N2bM0 \\
\hline 62 & M & 57 & Intermediate-grade MEC & Minor salivary gland & $8(5+3)$ & $6(3+3)$ & $94 \mathrm{R}(12)$ & pT1NxM0 \\
\hline 63 & M & 72 & Intermediate-grade MEC & Minor salivary gland & $7(4+3)$ & $6(3+3)$ & 31 & pT1NxM0 \\
\hline 64 & $\mathrm{~F}$ & 74 & Intermediate-grade MEC & Minor salivary gland & $6(3+3)$ & $6(3+3)$ & 95 & pT1NxM0 \\
\hline 65 & $\mathrm{~F}$ & 80 & Intermediate-grade MEC & Minor salivary gland & $6(3+3)$ & $6(3+3)$ & 97 & pT3NxM0 \\
\hline 66 & M & 26 & Intermediate-grade MEC & Parotid & $6(3+3)$ & $5(2+3)$ & 23 & pT1N0M0 \\
\hline 67 & M & 60 & Intermediate-grade MEC & Parotid & $6(3+3)$ & $5(2+3)$ & 34 & pT2N0M0 \\
\hline 68 & M & 50 & Intermediate-grade MEC & Minor salivary gland & $6(3+3)$ & $6(3+3)$ & 12 & pT1NxM0 \\
\hline 69 & M & 88 & Intermediate-grade MEC & Minor salivary gland & $7(4+3)$ & $6(3+3)$ & 12 & pT1NxM0 \\
\hline 70 & M & 77 & Intermediate-grade MEC & Minor salivary gland & $7(4+3)$ & $5(3+2)$ & 170 & pT3N0M0 \\
\hline 71 & M & 62 & Intermediate-grade MEC & Minor salivary gland & $7(4+3)$ & $5(3+2)$ & 45 & pT1NxM0 \\
\hline 72 & $\mathrm{~F}$ & 54 & Intermediate-grade MEC & Parotid & $6(3+3)$ & $5(3+2)$ & 12 & pT1NxM0 \\
\hline 73 & $\mathrm{~F}$ & 59 & Intermediate-grade MEC & Parotid & $6(3+3)$ & $5(3+2)$ & 14 & pT1NxM0 \\
\hline 74 & M & 56 & High-grade MEC & Minor salivary gland & $8(5+3)$ & $6(3+3)$ & $131 \mathrm{R}(75)$ & pT2N0M0 \\
\hline 75 & $\mathrm{~F}$ & 32 & High-grade MEC & Parotid & $7(4+3)$ & $5(2+3)$ & 200 & pT4NxM0 \\
\hline 76 & $\mathrm{~F}$ & 33 & High-grade MEC & Minor salivary gland & $7(4+3)$ & $5(2+3)$ & 23 & pT1NxM0 \\
\hline 77 & $\mathrm{~F}$ & 37 & High-grade MEC & Minor salivary gland & $6(3+3)$ & $6(3+3)$ & 21 & pT1NxM0 \\
\hline 78 & M & 46 & High-grade MEC & Minor salivary gland & $8(5+3)$ & $6(3+3)$ & $126 \mathrm{M}(13)$ & pT2N1M0 \\
\hline 79 & $\mathrm{~F}$ & 47 & High-grade MEC & Minor salivary gland & $7(4+3)$ & $6(3+3)$ & 23 & pT2NxM0 \\
\hline 80 & M & 51 & High-grade MEC & Sublingual gland & $7(4+3)$ & $6(3+3)$ & 72 & pT2N0M0 \\
\hline 81 & $\mathrm{~F}$ & 58 & High-grade MEC & Parotid & $8(5+3)$ & $6(3+3)$ & 39 M (2) & pT3N2M0 \\
\hline 82 & M & 60 & High-grade MEC & Submandibolar gland & $6(3+3)$ & $6(3+3)$ & 117 & pT3NxM0 \\
\hline 83 & $\mathrm{~F}$ & 41 & High-grade MEC & Minor salivary gland & $7(4+3)$ & $6(3+3)$ & 12 & pT1NxM0 \\
\hline 84 & $\mathrm{~F}$ & 78 & High-grade MEC & Sublingual gland & $7(4+3)$ & $6(3+3)$ & 142 & pT2NxM0 \\
\hline 85 & $\mathrm{~F}$ & 27 & High-grade MEC & Minor salivary gland & $7(4+3)$ & $6(3+3)$ & 155 & pT1NxM0 \\
\hline 86 & M & 60 & High-grade MEC & Parotid & $6(3+3)$ & $6(3+3)$ & 34 & pT3N0M0 \\
\hline 87 & $\mathrm{~F}$ & 56 & CXPA & Minor salivary gland & $7(4+3)$ & $6(3+3)$ & 23 & pT2NxM0 \\
\hline 88 & M & 19 & CXPA & Sublingual gland & $7(4+3)$ & $7(4+3)$ & 26 & pT2N0M0 \\
\hline 89 & $\mathrm{~F}$ & 24 & CXPA & Parotid & $7(4+3)$ & $7(4+3)$ & 97 & pT2N0M0 \\
\hline 90 & M & 42 & CXPA & Parotid & $8(5+3)$ & $7(4+3)$ & 55 R (10) N (10) & pT1N0M0 \\
\hline 91 & M & 43 & CXPA & Parotid & $6(3+3)$ & $7(4+3)$ & 63 & pT3N0M0 \\
\hline 92 & M & 72 & CXPA & Parotid & $7(4+3)$ & $6(3+3)$ & 166 & pT2NxM0 \\
\hline 93 & $\mathrm{~F}$ & 89 & CXPA & Parotid & $6(3+3)$ & $6(3+3)$ & 151 & pT3NxM0 \\
\hline 94 & M & 50 & CXPA & Parotid & $7(4+3)$ & $7(4+3)$ & 166 & pT2N0M0 \\
\hline
\end{tabular}

baseline benign tumour or the risk of an adverse event (recurrence or metastasis) in patients who had a baseline malignant tumour was calculated. Sensitivity and specificity of prognostic indices were computed according to standard formula. Receiver operating characteristic (ROC) curves were drawn and areas under the curve were calculated, to evaluate the discriminatory ability of each parameter, as follow: $<0.7$, no discrimination; 0.71-0.79, acceptable; 0.8-0.89, excellent; $\geq 0.9$, outstanding discrimination (27).

\section{Results}

The selected study population consisted of a cohort of 362 SGT (268 benign and 94 malignant).

Benign SGT. Benign tumours were composed of 265 PA, 4 of which with atypical/'bizarre' cells, and 3 cases of monomorphic (basal cell) adenomas. The clinical features of patients are reported in Table I. 


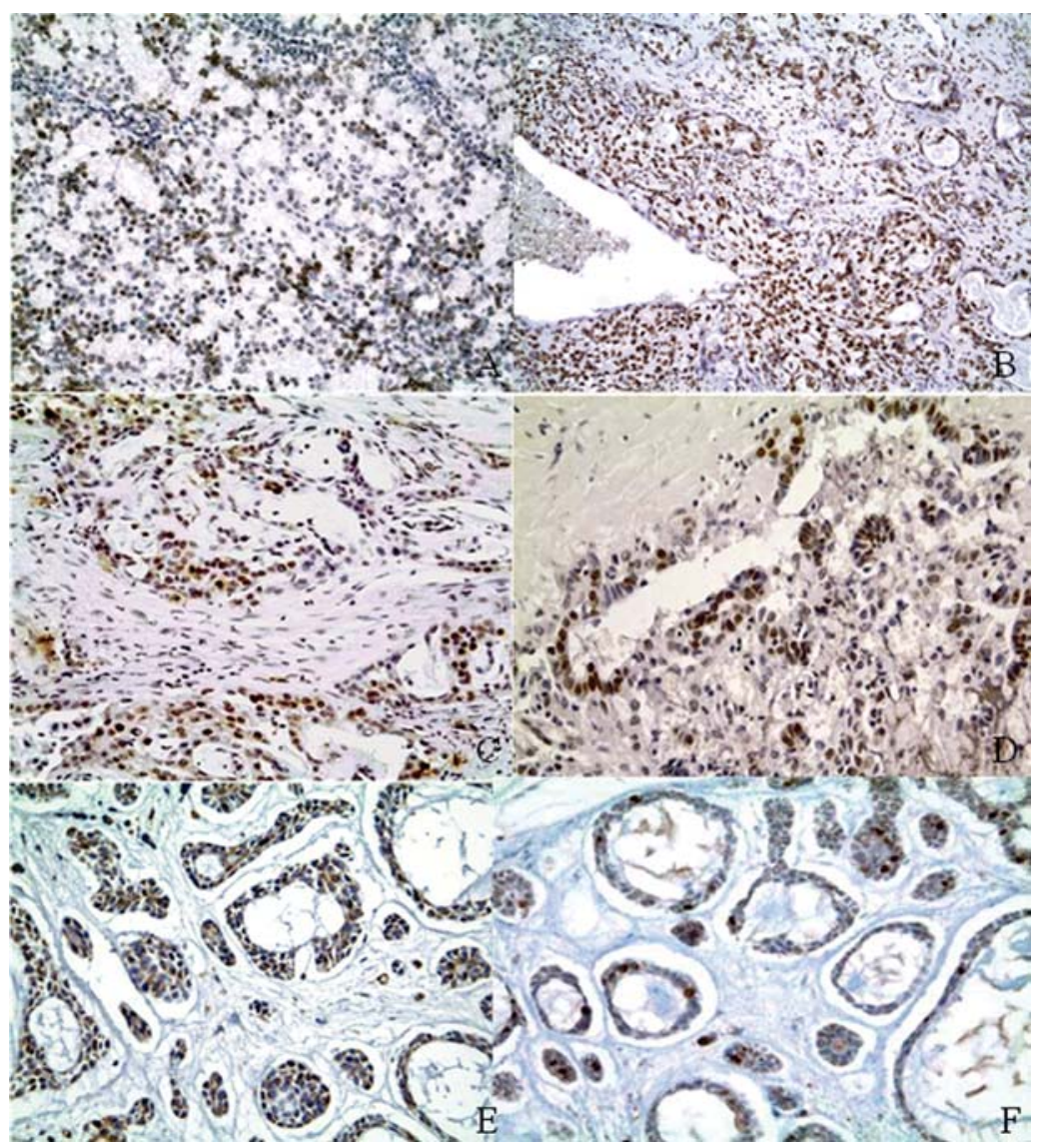

Figure 2. CAF-1/p60 overexpression in malignant SGT (LSAB-HRP, nuclear counterstaining with haematoxylin). Strong immunostaining for CAF-1/p60 in metastasizing malignant SGT: AC (A, x106), CXPA (B, x106), ME (C, x106), PLGA (D, x106). Metastasizing ACC: the same case showed strong expression for CAF-1/p60 (E, x150), and moderate expression for ki67/MIB1 (F, x150).

The cases for which PAP-stained slides were re-used for immunocytochemical evaluation of CAF-1/p60 were all PA (15 cases, parotid gland; 5 cases, submandibular gland; 3 cases, minor salivary glands, Fig. 1G).

During follow-up (mean 100.02 months, median 90.5 months, range 13-203 months), 5 patients (1.9\%) developed a CXPA. In three cases, the baseline tumour was a PA of the parotid gland, and in the remaining two cases atypical PA of minor salivary glands.

Malignant SGT. The malignant SGT consisted of 21 cases of adenoid cystic carcinomas (ACC), 2 polymorphous-low grade adenocarcinomas (PLGC), 14 acinic cell carcinomas (AC), 49 muco-epidermoid carcinomas (MEC: 22 low-grade, 14 intermediate-grade and 13 high-grade tumours) and 8 cases of carcinoma ex-PA (CXPA). The clinical features of all the tumours are reported in Table II.

During follow-up (mean 92.56 months, median 92.5 months, range $12-200$ months) 6 patients $(6.38 \%)$ had recurrence of the tumour (1PLGC, $1 \mathrm{AC}, 1 \mathrm{ACC}, 3 \mathrm{MEC}), 1$ $(1.06 \%)$ recurrence and nodal metastasis (1CXPA), 1 (1.06\%) recurrence and distant metastasis (1ACC) and $3(3.19 \%)$ distant metastasis (3 MEC).

\section{Immunohistochemistry}

$C A F-1 / p 60$ expression in benign SGT. The large majority of benign SGT (264 cases, 98.51\%) showed a quickscore 5 of immunostaining for CAF-1/p60 (Fig. 1A and B). Only 4 cases (1.55\%; all PA, two of the ordinary type and two atypical), which showed malignant transformation (CXPA) during follow-up, presented instead a quickscore 6 (Fig. 1C-F) (Table I).

The cytological smears concerned cases of PA which showed, on histological sections, a quickscore 5 in 20 cases and a score 6 in 3 cases which progressed to CXPA (Fig. 1G). Immunocytochemistry showed clusters of neoplastic cells strongly positive for the protein only in these 3 cases (Fig. 1H).

ki67/MIB1 expression in benign SGT. In the majority of benign SGT (231 out 268, 86.20\%), a quickscore 3 of ki67/MIB1 expression was found; 20 cases $(7.46 \%)$ showed a quickscore 4 and the remaining 17 cases $(6.34 \%)$ a quickscore 5 .

Among the cases which progressed toward malignancy during the follow-up, the quickscore was, respectively: 3 in 1 case, 4 in 2 cases and 5 in 2 cases (Table I).

$C A F-1 / p 60$ expression in malignant SGT. Overall, the quickscore for CAF-1/p60 expression ranged from 6 to 9 in malignant SGT (6-8 in AC, 6-9 in ACC, 6-7 in low-grade MEC, 6-9 in intermediate-grade MEC, 6-8 in high grade MEC, CXPA and PLGA).

The cases that recurred or progressed to nodal and/or distant metastases, showed always a quickscore 8-9 for CAF-1/p60 (Table II, Fig. 2A-E). 
ki67/MIB1 expression in malignant SGT. The quickscore for ki67/MIB1 in malignant SGT ranged from 4 to 7 (AC: quickscore 4; ACC: 4-6; low-grade MEC: 4-5; intermediateand high-grade MEC: 5-6; CXPA: 6-7; PLGC: 3). The recurrent and/or metastasizing cases showed a quickscore between 3 and 7 (Table II, Fig. 2F).

Expression of CAF-1/p60 and ki67/MIB1 in normal salivary glands. The residual normal salivary glands surrounding either benign and malignant SGT showed a quickscore 2 for ki67/MIB1 and 2-3 for CAF-1/p60.

\section{Statistical analysis}

$K$-statistics. The level of agreement of the evaluation of the quickscore of both CAF-1/p60 and ki67/MIB1, expressed by the K-coefficient, was 0.8 (excellent agreement) for both intra- and inter-observer evaluations, respectively.

Factors predicting development of cancer in patients with benign tumour baseline. According to univariate analysis, cancer onset was significantly related to: ki67/MIB1 expression $(\mathrm{p}<0.0001)$ and CAF-1/P60 $(\mathrm{p}<0.0001)$. Cox's multiple regression analysis selected $\mathrm{CAF}-1 / \mathrm{p} 60$ expression as the best independent and significant predictor of cancer development $(\mathrm{p}<0.0001)$.

Factors predicting disease-free intervals in patients with malignant tumour baseline. According to univariate analysis, the recurrence of tumour was significantly related to: female gender $(\mathrm{p}<0.05)$, ki67/MIB1 $(\mathrm{p}<0.0002)$ and CAF-1/p60 $(\mathrm{p}<0.0001)$. Applying Cox's multiple regression analysis, CAF-1/p60 expression $(\mathrm{p}<0.002)$ and staging $(\mathrm{p}<0.001)$ were the best set of covariates significantly predicting recurrence of the disease.

Concerning the occurrence of distant metastases, the univariate analysis showed that the expression of ki67/MIB1 $(\mathrm{p}<0.001), \mathrm{CAF}-1 / \mathrm{p} 60(\mathrm{p}<0.0001)$ and staging $(\mathrm{p}<0.0001)$ were significantly related to the metastasis development. Cox's multiple regression analysis selected CAF-1/p60 as the best independent predictor of metastasis onset $(\mathrm{p}<0.0004)$.

Clinical usefulness of $C A F-1 / p 60$ index. To further evaluate the usefulness of the CAF-1/p60 expression in the prediction of cancer development in patients who had a baseline benign tumour, sensitivity and specificity were calculated for each point of the quickscore, and receiver operating characteristic (ROC) curves were drawn. The best discriminant point of CAF-1/p60 quickscore was 5 , which had $80 \%$ sensitivity and $100 \%$ specificity, and the calculated area under curve corresponded to 0.9 (Fig. 3).

A similar analysis was performed to evaluate the usefulness of CAF-1/p60 in the prediction of an adverse event (recurrence, or nodal or distant metastasis) in patients who had a baseline malignant tumour. Sensitivity and specificity in predicting both recurrence of disease and metastasis, were calculated for each point of the quickscore, and ROC curves were drawn. The best discriminative point of CAF-1/p60 quickscore predicting both recurrence of disease or metastasis risk was 7 , which showed $100 \%$ sensitivity and $93 \%$ specificity in predicting recurrence of a tumour, and $100 \%$
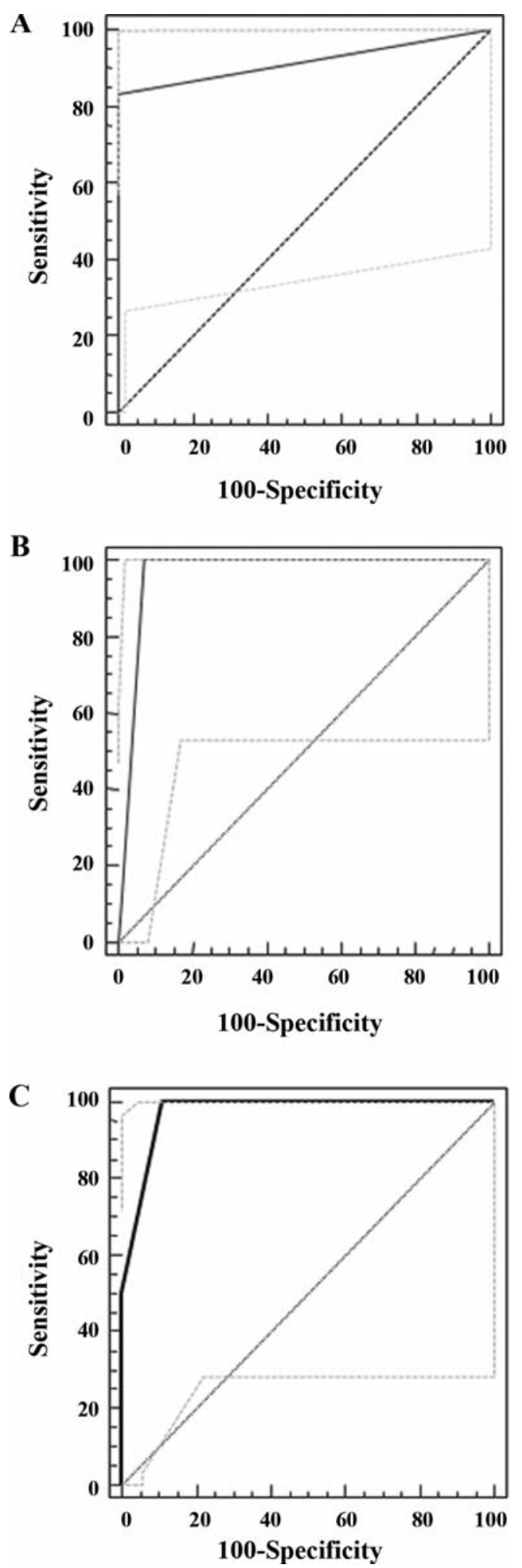

Figure 3. Receiver operating characteristic curves: (A) prediction of cancer development according to CAF-1/p60 immunopattern; (B) prediction of disease recurrence according to $\mathrm{CAF}-1 / \mathrm{p} 60$ immunopattern; (C) prediction of metastasis occurrence according to CAF-1/p60 immunopattern.

sensitivity and $89 \%$ specificity in predicting metastasis. Calculated areas under curve were 0.96 for the prediction of disease recurrence and 0.97 for prediction of metastasis, respectively (Fig. 3). 


\section{Discussion}

Salivary gland neoplasms comprise the most heterogeneous group of tumours of any body site, with frequent overlap of histological parameters and biological outcome $(1,2,28,29)$. Efforts to identify biomarkers able to assist in the differential diagnosis of these tumours and in predicting their evolution did not provide conclusive results (29-31).

Recent studies evidenced that epigenetic alterations may play a role in the development and progression of SGT (32-35). However, a relatively limited variety of SGT histotypes and genes analyzed for epigenetic regulation has been examined (36). The assembly and remodelling of chromatin are among the most important epigenetic modifications, in eukaryotic cells (37).

CAF-1 promotes the first step of nucleosome assembly in replicating DNA. The expression of CAF-1/p60 subunit directly correlates with cell proliferation in normal and neoplastic cells. This seems particularly attractive for researchers facing with SGT, considering that cell proliferation is one of the few proposed prognostic parameters for most of these tumours. The large majority of reports on this topic concern the immunohistochemical evaluation of the proliferating cell compartment by the ki67/MIB1 antibody.

The methodological approaches to this evaluation differed considerably among researchers. In particular, the evaluated ki67/MIB1 stained cells ranged from 500 to 1000 , randomly scored either with or without the aid of a video-scaler, or from the densest area of immunopositive cancer cells for $\mathrm{mm}^{2}$ of tumour tissue $(4,11,12)$.

These discrepancies and the frequently small retrospective series studied, due to the rarity of SGT, may explain the questionable results concerning the clinical value of ki67/ MIB1-stained cell fraction in evaluating the biological behaviour of SGT.

A correlation between a high ki67/MIB1 labelling index and the unfavourable prognosis of MEC has been reported, but for ACC some studies found ki67/MIB1 useful for predicting the biologic behaviour and/or treatment failure, while others found it not associated with morphology or clinical course of patients $(6,10,11,38)$.

Moreover, for AC, a study showed that most patients with tumour ki67/MIB1 indices above 10\% had unfavourable outcomes, whereas other reports evidenced overlap of indices between favourable and worse outcomes or showed that ki67/MIB1 revealed of statistical value in predicting tumour outcome $(7,39)$.

For PLGA, the ki67/MIB1 index was generally not found useful in predicting clinical behaviour and, in particular, Lazzaro and Cleveland showed that for PA, PLGA and ACC, the ki67/MIB1 score is not related either to the benign or malignant nature of tumours or to their specific clinical course (6,40-42).

For benign SGT, it has been stated that a moderate mitotic activity and older patient age, large tumour size and the occurrence in the submandibular gland, are associated to a greater likelihood of malignant transformation (43). However, a recent study evidenced that proliferation markers did not reveal significant differences between morpho- logically benign metastasizing PA and a control group of conventional PA (44).

Finally, the ki67/MIB1 index was thought either of overall utility in predicting the clinical behaviour of SGT, or it was considered significant only when coupled to TUNEL evaluation and p53 staining $(4,12,45)$.

Therefore, as it has recently emerged also for other malignant tumours, unresolved questions concerning the role of ki67/MIB1 as a real independent marker of prognosis for the major histotypes of malignant SGT still need to be answered.

Moreover, it has to be clarified whether this proliferation marker has the potential to detect early the cases of benign tumours which are trans-differentiating toward a malignant phenotype. It is still uncertain whether ki67/MIB1 is the best proliferation-associated predictor of malignancy and aggressiveness for SGT.

In this study, we decided to score an entire histological section of each SGT for ki67/MIB1 and CAF-1/p60 expression, evaluating them according to the 'quickscore' method, which is easy to perform by pathologists in addition to the routine diagnostic analysis. We found CAF-1/ p60 always expressed, in benign as in malignant SGT, with the highest degree of overexpression in more aggressive cases.

This was in-line with the reported association between the hyper-expression of the protein and the deregulation of the cell proliferation in malignant tumours of breast, tongue and prostate and in malignant melanoma, and was extremely significant in terms of statistical evaluation, besides tumour histotype, grade and stage at diagnosis $(18,20)$.

The overexpression of CAF-1/p60 may be then considered an overall hallmark of malignancy for SGT, which may assist in the challenging differential diagnosis between benign and malignant SGT. Moreover, the protein shows further significance in discriminating the cases of malignant SGT with metastatic ability.

Interesting considerations emerge also by the finding, in the majority of PA followed by the occurrence of CXPA after surgery, of a CAF-1/p60 quickscore higher than in nonprogressed benign SGT, with aggregates of neoplastic cells strongly positive for CAF-1/p60 in the corresponding FNAB preoperative samples of some of these cases.

In our opinion, CAF-1/p60 is highly effective to early identify the cases of benign epithelial salivary gland tumours which are trans-differentiating toward a malignant phenotype. This sounds particularly attractive for the evaluation of the biological behaviour of the clinically benign PA with 'bizarre/atypical' features, which sometimes may show an aggressive biological course, and of even greater importance for the cases of the classical form of PA, which rarely may evolve toward malignancy, in a manner unpredictable upon the classical histological and cytological parameters (46-48).

To date, this is the first study addressing the role of the expression of CAF-1/p60 in predicting the outcome of patients with SGT.

Considering the rarity of these tumours, we believe that our single institution's series of cases, for number, histotype of lesions and length of follow-up, may be considered adequate to evaluate the biological significance of the expression of CAF-1/p60 in SGT. 
Our results emphasize the role of epigenetic changes in SGT, and evidence a pivotal role for CAF-1/p60 as a marker of aggressiveness, with an additional value with respect to ki67/MIB1 in predicting the outcome of malignant SGT and improving the chances of early diagnosis of the subclass of benign SGT trans-differentiating toward malignancy. The utilization of the quickscore takes about 15 min per case, and appears of potential great utility for routine use for pathologists. Moreover, the appreciable immunostaining for the protein on FNAB samples, gives an additional chance of a preoperative 'biological' screening of patients with PA.

For therapeutic purposes, it has to be remembered that, up to date, neither conventional chemotherapy, nor the existing targeted drugs have shown satisfactory results against these tumours (49). The ultimate significance of our results in biological terms still needs to be definitely understood. Nevertheless, they encourage us to hypothesize a role for CAF-1/p60 as a promising molecular target for therapies tailored to reverse this epigenetic alteration in aggressive SGT.

\section{Acknowledgements}

We thank Ms. Amanda Tedeschi for the editing of the English of the manuscript, Maria Di Benedetto, BD, for her constructive observations and technical assistance, and Antonino Iaccarino, BTD, $\mathrm{PhDs}$, for his precious technical assistance for immunocytology. This study was funded by the Italian Ministry of Scientific and Technological Research (M.I.U.R.) Project of National Relevance (P.R.I.N.) No. 2007YMS5NS.

\section{References}

1. Eveson JV, Auclair P, Gnepp DR and El-Naggar AK: Tumors of the salivary glands: introduction. In: World Health Organization Classification of Tumours; Pathology and Genetics of Head and Neck Tumours. Barnes L, Everson JW, Reichart P and Sidrandky D (eds). IARC Press, Lyons, pp212-215, 2005.

2. Cheuk W and Chan JKC: Salivary glands tumors. In: Diagnostic Histopathology of Tumors. Fletcher CDM (ed). Livingstone, Churchill, pp239-326, 2007.

3. Cheuk W and Chan JK: Advances in salivary gland pathology. Histopathology 51: 1-20, 2007.

4. Ben-Izhak O, Laster Z, Araidy S and Nagler MR: TUNEL - an efficient prognosis predictor of salivary malignancies. Br J Cancer 96: 1101-1106, 2007.

5. Skálová A, Stárek I, Vanecek T, et al: Expression of HER-2/neu gene and protein in salivary duct carcinomas of parotid gland as revealed by fluorescence in-situ hybridization and immunohistochemistry. Histopathology 42: 348-356, 2003.

6. Luukkaa H, Klemi P and Leivo I: Prognostic significance of $\mathrm{Ki}-67$ and p53 as tumour markers in salivary gland malignancies in Finland: an evaluation of 212 cases. Acta Oncol 5: 669-675, 2006.

7. Skalova A, Leivo I, von Boguslawsky K and Saksela E: Cell proliferation correlates with prognosis in acinic cell carcinomas in salivary gland origin. Immunohistochemical study of 30 cases using the MIB 1 antibody in formalin-fixed paraffin sections. J Pathol 173: 13-21, 1994.

8. Skalova A, Lehtonen H, von Boguslawsky K and Leivo I: Prognostic significance of cell proliferation in mucoepidermoid carcinomas of the salivary gland. Clinicopathological study using MIB 1 antibody in paraffin sections. Hum Pathol 25: 929-935, 1994

9. Hellquist HB, Sundelin K, Di Bacco A, Tytor M, Manzotti M and Viale G: Tumour growth fraction and apoptosis in salivary gland acinic cell carcinomas. Prognostic implications of Ki-67 and bcl-2 expression and of in situ end labelling (TUNEL). J Pathol 181: 323-329, 1997.
10. Nordgard S, Franzen G, Boysen M and Halvorsen TB: Ki-67 as a prognostic marker in adenoid cystic carcinoma assessed with the monoclonal antibody MIB1 in paraffin sections. Laryngoscope 107: 531-536, 1997.

11. Kiyoshima T, Shima K, Kobayashi I, et al: Expression of p53 tumor suppressor gene in adenoid cystic and mucoepidermoid carcinomas of the salivary glands. Oral Oncol 37: 315-322, 2001.

12. Xin W and Paulino AF: Prognostic factors in malignant mixed tumors of the salivary gland: correlation of immunohistochemical markers with histologic classification. Ann Diagn Pathol 6: 205-210, 2002

13. Mello JA and Almouzni G: The ins and outs of nucleosome assembly. Curr Opin Genet Dev 11: 136-141, 2001.

14. Smith S and Stillman B: Purification and characterization of CAF-1, a human cell factor required for chromatin assembly during DNA replication in vitro. Cell 58: 15-25, 1989.

15. Kaufman PD, Kobayashi R, Kessler N and Stillmann B: The p150 and p60 subunits of chromatin assembly factor 1: a molecular link between newly synthesized histones and DNA replication. Cell 81: 1105-1114, 1995.

16. Ridgway $P$ and Almouzni G: CAF-1 and the inheritance of chromatin states: at the crossroads of DNA replication and repair. J Cell Sci 113: 2647-2658, 2000.

17. Ye X, Franco AA, Santos H, Nelson DM, Kaufmann PD and Adams PD: Defective S phase chromatin assembly causes DNA damage, activation of the $\mathrm{S}$ phase checkpoint, and $\mathrm{S}$ phase arrest. Mol Cell 11: 341-351, 2003

18. Polo SE, Yheocharis SE, Klijaieko J, et al: Chromatin assembly factor-1, a marker of clinical value to distinguish quiescent from proliferative cells. Cancer Res 64: 2371-2381, 2004.

19. Staibano S, Mignogna C, Lo Muzio L, et al: Chromatin assembly factor-1 (CAF-1) mediated regulation of cell proliferation and DNA repair: a link with the biological behaviour of squamous cell carcinoma of the tongue? Histopathology 50: 911-919, 2007.

20. Staibano S, Mascolo M, Mancini FP, et al: Overexpression of chromatin assembly factor-1 (CAF-1) p60 is predictive of adverse behaviour of prostatic cancer. Histopathology 54: 580-589, 2009.

21. Salivary glands. In: World Health Organization Classification of Tumours. Pathology and Genetics of Head and Neck Tumours. Barnes L, Everson JW, Reichart P and Sidrandky D (eds). IARC Press, Lyons, pp209-282, 2005.

22. Major salivary glands (parotid, submandibular, and sublingual). In: American Joint Committee on Cancer: AJCC Cancer Staging Manual. Greene FL, Page DL and Fleming ID (eds). 7th edition. Springer, New York, NY, pp103-109, 2010.

23. Jalava P, Kuopio T and Juntti-Patinen L: Ki67 immunohistochemistry: a valuable marker in prognostication but with a risk of misclassification: proliferation subgroups formed based on KI67 immunoreactivity and standardized mitotic index. Histopathology 48: 674-682, 2006.

24. Detre S, Saclani Jotti G and Dowsett M: A 'quickscore' method for immunohistochemical semiquantitation: validation for oestrogen receptor in breast carcinomas. J Clin Pathol 48: 876-878, 1995 .

25. Weintraub J, Redard M, Wenger D and Vassilakos P: The application of immunocytochemical technique to routinely fixed and stained cytologic specimens. An aid in the differential diagnosis of undifferentiated malignant neoplasms. Pathol Res Pract 186: 658-665, 1990.

26. Landis JR and Koch GG: The measurement of observer agreement for categorical data. Biometrics 33: 159-174, 1997.

27. Zweigh MH and Campbell G: Receiver operating characteristic (ROC) plats: a fundamental evaluation tool in clinical medicine. Clin Chem 39: 561-577, 1993.

28. Brandwein MS, Ferlito A, Bradley PJ, Hille JJ and Rinaldo A: Diagnosis and classification of salivary neoplasms: pathologic challenges and relevance to clinical outcome. Acta Otolaryngol 122: 758-764, 2002.

29. El-Naggar AK, Dinh M, Tucker Sl, Gillenwater A, Luna MA and Batsakis JG: Chromosomal and DNA ploidy characterization of salivary gland neoplasms by combined FISH and flow cytometry. Hum Pathol 28: 881-886, 1997.

30. El-Naggar AK, Hurr K, Kagan J and Batsakis JG: Genotypic alterations in benign and malignant salivary gland tumour: histogenetic and clinical implications. Am J Surg Pathol 21: 691-697, 1997. 
31. Edwards PC, Bhuiya T and Kelsch RD: C-kit expression in the salivary gland neoplasms adenoid cystic carcinoma, polymorphous low-grade adenocarcinoma, and monomorphic adenoma. Oral Surg Oral Med Oral Pathol Oral Radiol Endod 95: 586-593, 2003.

32. Gatta G, Ciccolallo L, Kunkler I, et al: Survival from rare case in adults: a population-based study. Lancet Oncol 7: 132-140, 2006.

33. Uro-Coste E: Tumeurs des glandes salivaires. E'tat des lieux en 2009-2009 update in salivary gland tumoral pathology. Ann Pathol 29: 274-285, 2009.

34. Williams MD, Chakravarti N, Kies MS, et al: Implications of methylation patterns of cancer genes in salivary gland tumors. Clin Cancer Res 12: 7353-7358, 2006.

35. Kishi M, Nakamura M, Nishimine M, Ikuta M, Kirita T and Konishi N: Genetic and epigenetic alteration profiles for multiple genes in salivary gland carcinomas. Oral Oncol 41: 161-169, 2005.

36. Maruya S, Kim HW, Weber RS, et al: Gene expression screening of salivary gland neoplasms: molecular markers of potential histogenetic and clinical significance. J Mol Diagn 6: 180-190, 2004 .

37. Polo SE and Almouzni G: Chromatin assembly: a basic recipe with various flavours. Curr Opin Genet Dev 16: 104-111, 2006.

38. Norberg-Spaak L, Dardick I and Ledin T: Adenoid cystic carcinoma: use of cell proliferation, BCL-2 expression, histologic grade, and clinical stage as predictors of clinical outcome. Head Neck 22: 489-497, 2000.

39. Michael M, Skalova A, Simpson RH, Laivo I, Ryska A and Starek I: Well-differentiated acinic cell carcinoma of salivary glands associated with lymphoid stroma. Hum Pathol 28: 595-600, 1997

40. Perez-Ordonez B, Linkov I and Huvos AG: Polymorphous lowgrade adenocarcinoma of minor salivary glands: a study of 17 cases with emphasis on cell differentiation. Histopathology 32 : $521-529,1998$
41. Lazzaro B and Cleveland D: P53 and Ki-67 antigen expression in small oral biopsy specimens of salivary gland tumors. Oral Surg Oral Med Oral Pathol Oral Radiol Endod 89: 613-617, 2000.

42. Zhao M, Zhao QX, Saitoh M, et al: Proliferating patterns of salivary gland adenocarcinomas with the use of PCNA labeling. Anticancer Res 16: 2693-2698, 1996.

43. Auclair PL and Ellis GL: Atypical features in salivary gland mixed tumors: their relationship to malignant transformation. Mod Pathol 9: 652-657, 1996.

44. Marioni G, Marino F, Stramare R, Marchese-Ragona R and Staffieri A: Benign metastasizing pleomorphic adenoma of the parotid gland: a clinicopathologic puzzle. Head Neck 25: 1071-1076, 2003.

45. Spyratos F, Ferrero-Poüs M, Trassard M, et al: Correlation between MIB-1 and other proliferation markers: clinical implications of the MIB-1 cutoff value. Cancer 94: 2151-2159, 2002.

46. Eveson JV, Kasafuka K, Stenman G and Nagao T: Pleomorphic adenoma. In: World Health Organization Classification of Tumours. Pathology and Genetics of Head and Neck Tumours. Barnes L, Everson JW, Reichart P and Sidrandky D (eds). IARC Press, Lyons, pp254-258, 2005.

47. LiVolsi VA and Perzin KH: Malignant mixed tumours arising in salivary glands. I. Carcinomas arising in benign mixed tumors: a clinicopathologic study. Cancer 39: 2209-2230, 1977.

48. Altemani A, Martins MT, Freitas L, Soares F, Araujo NS and Araujo VC: Carcinoma ex pleomorphic adenoma (CXPA): immunoprofile of the cells involved in carcinomatous progression. Histopathology 46: 635-641, 2005.

49. Milano A, Longo F, Basile M, Iaffaioli RV and Caponigro F: Recent advances in the treatment of salivary gland cancers: emphasis on molecular targeted therapy. Oral Oncol 43: 729-34, 2007. 